\title{
The Applications and Practice of Additional Workpiece Coordinate System (G54.1 $\left.P_{n}\right)$
}

\author{
Zhengrong Zhou ${ }^{1, a}$ \\ ${ }^{1}$ Engineering Training Center, Guizhou University, China
}

\begin{abstract}
The choice of the workpiece coordinate system is a very important for machining center programming practice. Additional coordinate system is often neglected because of "addition", but under the condition of processing multiple parts at the same time, the programming quality and the efficiency of programming can be greatly improved. This paper is present with the additional workpiece coordinate system of FANUC NC system with application examples, which provide the reference for colleague.
\end{abstract}

\section{INTRODUCTION}

The machining center of FANUC NC system can be seen everywhere in the field of machining. Personnel are dreamed of skilled operation on course of machining center. In general, the program can be used by manual or automatic or combination of both after parts processing technology is established. No matter which method, the program can be compiled with availability, good and fast. "Availability" is the quality and "good" is the sublimation and improvement, but "fast" is the efficiency. Personnel engaged in the industry constantly learning, exploration, innovation for better NC program which is endless power. This paper is present with additional workpiece coordinate system (G54.1 Pn) in $\mathrm{NC}$ programming based on FANUC NC system. Processing of multiple parts process is different from general and worthy of promotion with some examples.

\section{What is the workpiece coordinate system}

\subsection{The concept of the workpiece coordinate system}

The workpiece coordinate system refers to the process to determine the origin coordinate system was established by the benchmark. The origin, the origin of the machining center, also known as procedure is refers to the parts packed clip, the corresponding programming origin position in machine coordinate system. In the machining process, nc machine tools is according to the workpiece clamping after processing the origin as determined in the position and application requirement for processing. Programmers in the programming, as long as according to part drawing can be selected programming the origin, establishing programming coordinate, coordinate numerical calculation, without considering the workpiece clamping the actual position of the blank. For processing personnel, nc machine tools should be in the clamping workpiece, debugging program, converts programming origin to processing the origin, and determine the position of the processing origin, processing center in a $\mathrm{CNC}$ system for setting value (that is, given the source), yet after the workpiece coordinate system can be based on the current tool position, determine the coordinates of tool start point. When processing, the size of the workpiece coordinate values are relative to the case of processing origin, in this way can $\mathrm{CNC}$ machine according to the accurate position of the workpiece coordinate system began to processing.

\subsection{The principles of choosing workpiece coordinate system}

Workpiece zero elected in part on the size of the benchmark, so to facilitate the calculation of coordinates, and reduce errors.

The workpiece zero point try to choose in high precision of workpiece surface, in order to improve the machining precision of processed parts.

For symmetrical parts, the workpiece zero point is located in the center of symmetry.

For general parts, the workpiece zero point is located in the workpiece contour on the corner.

The $\mathrm{z}$ axis direction zero generally in the workpiece surface.

When programming, cutting tool should be the starting point and the procedure the origin in the same place, it can simplify the procedure, easy to calculate.

\subsection{A common way to determine the workpiece coordinate system}

Using G54 G59 choice workpiece coordinate system.

\footnotetext{
${ }^{\mathrm{a}}$ Corresponding author: ig.zrzhou@gzu.edu.cn
} 
Using G54, - G59 instructions can choose a in default of the workpiece coordinate system as the workpiece coordinate system. The coordinates of the six workpiece coordinate system origin coordinate values in the machine tool coordinate system (called zero offset value), must be before the program is running, from "zero offset" input interface. More commonly used in the need to build more than one workpiece coordinate system. Choose good after the workpiece coordinate system, if the replacement tool, cutting tool length compensation is combined with instructions to coordinate transform $\mathrm{Z}$ can, don't need to replace the workpiece coordinate system.

Using G92 set workpiece coordinate system.

Using G92 set workpiece coordinate system for CNC milling machine and machining center. Using the instruction of the workpiece coordinate system origin in relative to the cutting tool starting point can be set on a space to meet the requirements of processing point. G92 have strict request for the starting point of the tool, if the cutting tool, the starting point of the current point is not set by G92, processing the origin and application do not match the origin, processing of the products is the error or scrapped, appear even dangerous. When performing the directive, therefore cutting tool the current point must be just on the knife point namely G92 coordinate values specified by the workpiece coordinate system. Found in the execution of cutter operation after the workpiece coordinate origin, also need more than one step positioning tool to program the operation of the starting point, more trouble.

Additional workpiece coordinate system ( G54.1 Pn) G54.1 P1, G54.1 P2, G54.1P3... G54.1 P48, can set up 48 workpiece coordinate system at the same time.

\section{Application examples on additional workpiece coordinate system}

When carring on the machining center $\mathrm{CNC}$ programming we will generally choose standard coordinate system establishment from G54 - G59 of one or a few, which are not more than six. But when need to choose more than six workpiece coordinate system, how to do? At this time, additional workpiece coordinate system can be used and very convenient.

For FANUC NC system, additional work coordinate system refers to the G54.1 Pn, where $\mathrm{n}$ is a integer from 1 to 48.It is enough to use because it can improve the choice of workpiece coordinate system number to 48 under normal circumstances . Because of additional work coordinate system and standard work coordinate system set in the same way, this should not be detailed here. Next we introduce the method of programming used additional coordinate system in $\mathrm{CNC}$ through parts processing flow chart shown in figure 1 (fourteen parts one time).

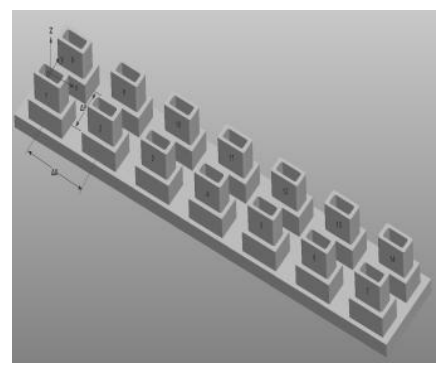

Figure 1 a parts processing process diagram

The first step, according to the process diagram develop process.

Can be seen from the diagram process, need to work out the shape and inner cavity of the 14 parts through the clamping at a time.Assume that parts of $x, y, z$ direction of the maximum size of $20 \mathrm{~mm}, 10 \mathrm{~mm}, 8 \mathrm{~mm}$. Here, we use a combination of manual and automatic programming for programming. The inner cavity of the machining method can consult appearance.If we choose to use $8 \mathrm{~mm}$ diameter vertical milling cutter for machining shape, by simple calculation, the Blank materialdimension of at least $118 \mathrm{~mm}(7 * 10+8 * 6) *(2 * 10+8) 28 * 12 \mathrm{~mm}$ (assuming that the minimum is $3 \mathrm{~mm}$ ). Here, we according to the experience of the processing, we chose $120 * 30 * 13$.

The second step, the establishment of parts programming coordinate system, as shown in figure 1 (Coordinate system origin in the first part on the center of the surface)

The third step, the components of the program (including the main program and subroutine), and the main program using manual writing, compiled subroutines using MasterCAM X4 (after the simulation effect is shown in figure 2). Procedure is as follows:

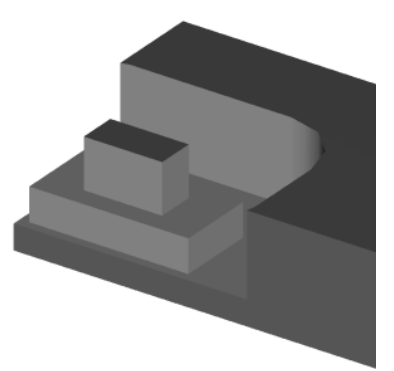

Figure 2 parts processing simulation diagram

O8800

$(\mathrm{sx}-001)$

N1

T1 M6

(D8R)

G40G80 S2000M13

G0 G90 G54.1P01 X0Y0

G43H1Z5

M98P8801F250

G0Z5.

G0G90G54.1P02X0Y0

M98P8801

G0Z5.

G0G90G54.1P03X0Y0 
M98P8801

G0Z5.

G0G90G54.1P04X0Y0

M98P8801

G0Z5.

G0G90G54.1P05X0Y0

M98P8801

G0Z5.

G0G90G54.1P06X0Y0

M98P8801

G0Z5.

G0G90G54.1P07X0Y0

M98P8801

G0Z5.

G0G90G54.1P08X0Y0

M98P8801

G0Z5.

G0G90G54.1P09X0Y0

M98P8801

G0Z5.

G0G90G54.1P10X0Y0

M98P8801

G0Z5.

G0G90G54.1P11X0Y0

M98P8801

G0Z5.

G0G90G54.1P12X0Y0

M98P8801

G0Z5.

G0G90G54.1P13X0Y0

M98P8801

G0Z5.

G0G90G54.1P14X0Y0

M98P8801

G0Z200.

G40

M5

M9

G91 G28 Y0.

M30

O8801

$\%$

G0X-10. Y-9. S2000 M13

Z

00 .

X10. F250.

G3 X14. Y-5. I0. J4.

G1 Y5.

G3 X10. Y9. I-4. J0.

G1 X-10. 25.

Z5.F800

G1 Z-2.5 F3

G3 X-14. Y5. I0. J-4.

G1 Y-5.

G3 X-10. Y-9. I4. J0.

G1 Z-5. F300.

$\mathrm{X} 10 . \mathrm{F} 250$.

G3 X14. Y-5. I0. J4.

G1 Y5.

G3 X10. Y9. I-4. J0.

G1 X-10.

G3 X-14. Y5. I0. J-4.
G1 Y-5.

G3 X-10. Y-9. I4. J0.

G1 Z-7.5 F300.

$\mathrm{X} 10$. F250.

G3 X14. Y-5. I0. J4.

G1 Y5.

G3 X10. Y9. I-4. J0.

G1 X-10.

G3 X-14. Y5. I0. J-4.

G1 Y-5.

G3 X-10. Y-9. I4. J0.

G1 Z-10. F300.

$\mathrm{X} 10$. F250.

G3 X14. Y-5. I0. J4.

G1 Y5.

G3 X10. Y9. I-4. J0.

G1 X-10.

G3 X-14. Y5. I0. J-4.

G1 Y-5.

G3 X-10. Y-9. I4. J0.

G0 Z25.

$\mathrm{X}-5$. Y-6.5

$\mathrm{Z5}$.

G1 Z-3. F300.

X5. F250.

G3 X9. Y-2.5 I0. J4.

G1 Y2.5

G3 X5. Y6.5 I-4. J0.

G1 X-5.

G3 X-9. Y2.5 I0. J-4.

G1 Y-2.5

G3 X-5. Y-6.5 I4. J0.

G1 Z-6. F300.

X5. F250.

G3 X9. Y-2.5 I0. J4.

G1 Y2.5

G3 X5. Y6.5 I-4. J0.

G1 X-5.

G3 X-9. Y2.5 I0. J-4.

G1 Y-2.5

G3 X-5. Y-6.5 I4. J0.

G0 Z25.

M99

$\%$

The fourth step, set G54.1 Pn workpiece coordinate system parameters. As follows, additional work coordinate system into the system interface, set the first part of the workpiece coordinate system G54.1 P01, then the corresponding input P02 to P14 data (see table 1)

After completion, the above four steps can be in accordance with the parts processing technology on a clamping completed 14 parts processing. The whole process is simple, quick, is not an efficient processing method. 
Table 1 additional workpiece coordinate system data table

\begin{tabular}{|c|c|c|c|c|}
\hline \multirow{2}{*}{$\begin{array}{c}\text { Additional } \\
\text { coordinate } \\
\text { system }\end{array}$} & \multicolumn{4}{|c|}{ Coordinate system data } \\
\cline { 2 - 5 } & $\mathrm{X}$ & $\mathrm{Y}$ & $\mathrm{Z}$ & $\mathrm{A}$ \\
\hline G54.1 P01 & 0 & 0 & 0 & 0 \\
\hline G54.1 P02 & $1 \Delta \mathrm{x}$ & 0 & 0 & 0 \\
\hline G54.1 P03 & $2 \Delta \mathrm{x}$ & 0 & 0 & 0 \\
\hline G54.1 P04 & $3 \Delta \mathrm{x}$ & 0 & 0 & 0 \\
\hline G54.1 P05 & $4 \Delta \mathrm{x}$ & 0 & 0 & 0 \\
\hline G54.1 P06 & $5 \Delta \mathrm{x}$ & 0 & 0 & 0 \\
\hline G54.1 P07 & $6 \Delta \mathrm{x}$ & 0 & 0 & 0 \\
\hline G54.1 P08 & 0 & $1 \Delta \mathrm{y}$ & 0 & 0 \\
\hline G54.1 P09 & $1 \Delta \mathrm{x}$ & $1 \Delta \mathrm{y}$ & 0 & 0 \\
\hline G54.1 P10 & $2 \Delta \mathrm{x}$ & $1 \Delta \mathrm{y}$ & 0 & 0 \\
\hline G54.1 P11 & $3 \Delta \mathrm{x}$ & $1 \Delta \mathrm{y}$ & 0 & 0 \\
\hline G54.1 P12 & $4 \Delta \mathrm{x}$ & $1 \Delta \mathrm{y}$ & 0 & 0 \\
\hline G54.1 P13 & $5 \Delta \mathrm{x}$ & $1 \Delta \mathrm{y}$ & 0 & 0 \\
\hline G54.1 P14 & $6 \Delta \mathrm{x}$ & $1 \Delta \mathrm{y}$ & 0 & 0 \\
\hline
\end{tabular}

The choice of the workpiece coordinate system and setting there are many kinds of methods, the specific choice of which method is different from person to person, owing to the different numerical control system, owing to the different components of the processing technology, reasonable choice it, not only can simplify the programming, the program is more important is easy to debug, easy to modify, easy to understand, this need to keep learning and exploration, in study practice, found in practice, innovation in the discovery.

\section{Conclusion}

The choice of the workpiece coordinate system and setting there are many kinds of methods, the specific choice of which method is different from person to person, owing to the different numerical control system, owing to the different components of the processing technology, reasonable choice it, not only can simplify the programming, the program is more important is easy to debug, easy to modify, easy to understand, this need to keep learning and exploration, in study practice, found in practice, innovation in the discovery.

\section{References}

1. FANUC Series 0 I - MODEL D user manual

2. CNC programming manual.(American) Smith, xueke luo et al.Chemical industry press.2012

3. Several methods of workpiece coordinate setting during. Rendong. Machine Tool \& Hydraulics. 2004 (1)

4. Fanuc 0i- MC system processing center coordinate system setup and applications. Baohua Sun, Zexun Zhang. Machinist Metal Cutting .10. 2009

5. To establish numerical control method of workpiece coordinate system review. Xinghong Qi, Xianwen Xiong Jianying Huang. Machine Design and Research, Vo. L 22 No. 2 apr.2006

6. CNC machining center the establishment of the workpiece coordinate system Xianwen, Xiong Xingzu Ming. Modular Machine Tool \& Automatic Manufacturing Technique 2003. (12)

7. Based on FANUC 0iM system of workpiece coordinate setting method research. Lifeng, Xiangdong. Coal Technology 2012 no. 2

8. Machining center used the setting of workpiece coordinate system and application. Jiaxiao liu. machinery $46(52)$ 